\title{
A nomogram based on pretreatment levels of serum bilirubin and total bile acid levels predicts survival in colorectal cancer patients
}

Yinghao Cao ${ }^{1 \dagger}$, Shenghe Deng ${ }^{1 \dagger}$, Lizhao Yan ${ }^{1 \dagger}$, Junnan Gu , Jia Yang ${ }^{2}$, Ming Yang ${ }^{3}$, Li Liu ${ }^{4^{*}}$ and Kailin Cai ${ }^{1 *}$

\begin{abstract}
Background: Serum bilirubin and total bile acid (TBA) levels have been reported to be strongly associated with the risk and prognosis of certain cancers. Here, we aimed to investigate the effects of pretreatment levels of serum bilirubin and bile acids on the prognosis of patients with colorectal cancer (CRC).

Methods: A retrospective cohort of 1474 patients with CRC who underwent surgical resection between January 2015 and December 2017 was included in the study. Survival analysis was used to evaluate the predictive value of pretreatment levels of bilirubin and bile acids. X-Tile software was used to identify optimal cut-off values for total bilirubin (TBIL), direct bilirubin (DBIL) and TBA in terms of overall survival (OS) and disease-free survival (DFS).

Results: DBIL, TBIL, and TBA were validated as significant prognostic factors by univariate Cox regression analysis for both 3-year OS and DFS. Multivariate Cox regression analyses confirmed that high DBIL, TBIL and TBA levels were independent prognostic factors for both OS (HR: 0.435, 95\% Cl: 0.299-0.637, $P<0.001$; HR: $0.436,95 \%$ Cl: $0.329-$ $0.578, P<0.001$; HR: $0.206,95 \%$ Cl: $0.124-0.341, P<0.001$, respectively) and DFS (HR: $0.583,95 \%$ Cl: $0.391-0.871, P=$ 0.008; HR:0.437,95\% Cl: 0.292-0.655, $P<0.001$; HR: $0.634,95 \%$ Cl: $0.465-0.865, P=0.004$, respectively). In addition, nomograms for OS and DFS were established according to all significant factors, and the c-indexes were 0.819 (95\% Cl: $0.806-0.832)$ and 0.835 (95\% Cl: 0.822-0.849), respectively.
\end{abstract}

Conclusions: TBIL, DBIL and TBA levels are independent prognostic factors in colorectal cancer patients. The nomograms based on OS and DFS can be used as a practical model for evaluating the prognosis of CRC patients.

Keywords: Colorectal cancer, Total bilirubin, Direct bilirubin, Total bile acid, Survival analysis

\footnotetext{
* Correspondence: gracefulliuly@163.com; caikailin@hust.edu.cn

${ }^{\dagger}$ Yinghao Cao, Shenghe Deng and Lizhao Yan contributed equally to this

work.

${ }^{4}$ Department of Epidemiology and Biostatistics, the Ministry of Education Key

Lab of Environment and Health, School of Public Health, Tongji Medical

College, Huazhong University of Science and Technology, Wuhan, Hubei

430022, China

'Department of Gastrointestinal Surgery, Union Hospital, Tongji Medical

College, Huazhong University of Science and Technology, 1277 JieFang Avenue, Wuhan 430022, Hubei, China

Full list of author information is available at the end of the article
}

C C The Author(s). 2021 Open Access This article is licensed under a Creative Commons Attribution 4.0 International License, which permits use, sharing, adaptation, distribution and reproduction in any medium or format, as long as you give appropriate credit to the original author(s) and the source, provide a link to the Creative Commons licence, and indicate if changes were made. The images or other third party material in this article are included in the article's Creative Commons licence, unless indicated otherwise in a credit line to the material. If material is not included in the article's Creative Commons licence and your intended use is not permitted by statutory regulation or exceeds the permitted use, you will need to obtain permission directly from the copyright holder. To view a copy of this licence, visit http://creativecommons.org/licenses/by/4.0/ The Creative Commons Public Domain Dedication waiver (http://creativecommons.org/publicdomain/zero/1.0/) applies to the data made available in this article, unless otherwise stated in a credit line to the data. 


\section{Background}

CRC ranked third among the three most common cancers in both males and females in 2019 and is one of the leading causes of cancer-related mortality worldwide. It is estimated that as of January 1, 2019, more than 1.5 million men and women in the United States had been diagnosed with colorectal cancer, and 145,600 new cases will be confirmed in 2019 [1]. With the improvement in living conditions and changes in dietary habits, the incidence of CRC has been increasing in China in recent years, and surgical resection is still the only treatment for CRC at present [2].

Predicting the long-term survival of patients with CRC can be challenging due to genetic, dietary, and geographical differences. However, accurate prediction of prognosis is essential for treatment selection and communication between doctors and patients. Previous studies have shown that age, lymph node status, systemic inflammation, perineural invasion, etc. risk factors can predict the survival rate of CRC patients after surgical resection, and the most important risk factor is the tumour-node-metastasis (TNM) staging system [3, 4]. However, CRC is a heterogeneous disease, and even when patients are at the same stage of disease, the prognosis varies. The current TNM staging system has the limitations of simplicity and unity and does not take into account some important variables that may affect CRC patient survival, including clinicopathological features and adjuvant therapy. Therefore, it cannot accurately predict the prognosis of CRC patients.

Serum bilirubin is the end product of haem metabolism and was once considered to have no physiological function, but the latest research has shown that it not only has many protective properties, including effective antioxidant, anti-inflammatory and anticancer activities, but is also negatively associated with the risk of a variety of cancers, including breast cancer, lung cancer, and CRC [5-8]. Additionally, abnormally high levels of TBA, which connect the intestinal microbiota with the liver and intestinal metabolism, trigger excessive harmful effects on the colonic mucosa, markedly promoting CRC progression [9]. Gao et al. suggested that increased direct serum bilirubin levels were associated with lymph node metastasis and poor prognosis in rectal cancer patients, and Zhang et al. used nomograms based on direct serum bilirubin levels to predict the prognosis of stage II and III CRC patients [5, 10]. Although considering these factors improves the prognosis of CRC, no measurement or indicator combining serum bilirubin and TBA levels has been developed. The purpose of this study was to evaluate the ability of combined pretreatment levels of serum bilirubin and TBA levels to predict survival outcomes in CRC patients after radical resection. In addition, we developed nomograms to evaluate the predictive value of pretreatment levels of serum bilirubin and TBA levels in these patients.

\section{Methods}

\section{Study design and patient selection}

A total of 1474 patients with colorectal cancer who underwent surgical resection at Wuhan Union Medical College Hospital between January 2015 and December 2017 were included in this study. The inclusion criteria were as follows: (1) age $>20$ years old with no preoperative antitumour therapy; (2) radical resection of primary CRC; (3) pathology confirming all stage I to IV patients; and (4) complete clinical and pathological data. Patients with the following conditions were excluded from the study: (1) colonic perforation and peritonitis; (2) history of tumour and death of other causes during the followup period; (3) severe cardiovascular disease; (4) patients with primary hepatobiliary disease whose serum bilirubin may be increased or decreased; and (5) other interventions, such as stent placement before radical surgery.

The following parameters were included in the analysis: age, sex, smoking status, tumour history, intestinal obstruction, tumour differentiation, tumour size, tumour location, tumour $\mathrm{T}$ stage, tumour $\mathrm{N}$ stage, tumour TNM stage, perineural invasion, vascular invasion, chemotherapy, TBIL, DBIL and TBA. All patients were reclassified according to the 7th edition of the American Joint Committee on Cancer (AJCC) TNM classification, and patients with stage II disease of high risk and/or above received adjuvant chemotherapy postoperatively.

Follow-up data were obtained every three months, and when we suspected recurrence, gastroscopy and imaging were performed at that visit time. Overall survival rate (OS) was defined as the time interval from surgery to death or the last follow-up. Disease-free survival (DFS) was defined from the date of definitive surgery to the date of first recurrence (local or distant) or date of last follow-up.

\section{Statistical analysis}

Statistical analysis was performed using SPSS 23.0 (SPSS Inc., Chicago, IL, USA) and R 4.0.0 software (Institute for Statistics and Mathematics, Vienna, Austria). X-tile 3.6.1 (Yale University, New Haven, CT, USA) was used to determine the optimal cut-off value for TBIL, DBIL, and TBA levels. The difference between the high and low level groups was evaluated by either the $\chi^{2}$ test or the mann-whitney $U$ test. The 3-year OS and DFS were estimated by the Kaplan-Meier method, and the difference of variables was compared using log-rank tests. Univariate and multivariate Cox proportional hazards regression models were used to evaluate the prognostic factors for OS and DFS by calculating hazard ratios and their 95\% CIs. A nomogram of the important factors related to the OS and DFS were constructed with $\mathrm{R}$ software, and the performance of the nomogram was evaluated by the Harrell consistency index (c-index). To 
further evaluate the accuracy of the nomogram in predicting prognosis, a calibration curve was generated comparing the observed results with the predicted results. A value of $P<0.05$ was considered significant.

\section{Results}

\section{Patient clinical characteristics}

A total of 1474 CRC patients were recruited for this study, including 867 males and 607 females. The mean age of patients was $57.99 \pm 12.26$ years (range, 20-85). The median values of TBIL, DBIL, and TBA were 7.6 (range 0.20-75.30) $\mu \mathrm{mol} / \mathrm{l}, 6.7$ (range 0.60-50.20) $\mu \mathrm{mol} /$ 1 , and 4.1 (range $0.20-86.20$ ) $\mu \mathrm{mol} / \mathrm{L}$, respectively.

\section{Associations among TBIL, DBIL and TBA levels and clinical characteristics}

Univariate Cox regression indicated that TBIL, DBIL and TBA (when they were continuous variables) were important prognostic factors for OS and DFS, and the
X-Tile program was used to determine the optimal cutoff values for DBIL in terms of OS and DFS. The optimal cut-off values were $6.4 \mu \mathrm{mol} / \mathrm{L}$ for TBIL, $12.8 \mu \mathrm{mol} /$ $\mathrm{L}$ for DBIL, and $7.1 \mu \mathrm{mol} / \mathrm{L}$ for TBA based on OS (Fig. 1), and the optimal cut-off values were $5.2 \mu \mathrm{mol} / \mathrm{L}$ for TBIL, $13.1 \mu \mathrm{mol} / \mathrm{L}$ for DBIL, and $6.8 \mu \mathrm{mol} / \mathrm{L}$ for TBA based on DFS (Fig. 2). Next, patients were divided into high and low groups according to the optimal cut-offs. Lymph node metastasis (stage N1 and N2) was more common in patients with high TBIL than in patients with low TBIL (44.9\% vs $37.4 \%, P=0.026)$, but the average tumour diameter was smaller in the high group than in the low group $(4.09 \pm 2.11$ vs $4.42 \pm 2.02, P=0.008)$ (Table 1). Compared to the low DBIL group, the high DBIL group had significantly more patients with stage III and IV disease $(58.3 \%$ vs $51.8 \%, P=0.022)$. In addition, males $(67.5 \%$ vs $57.7 \%, P=0.017)$, obstructions (22.1\% vs $15.4 \%, P=0.029)$, and smaller tumour diameters $(3.73 \pm 1.76$ vs $4.25 \pm 2.12, P=0.003)$ were more
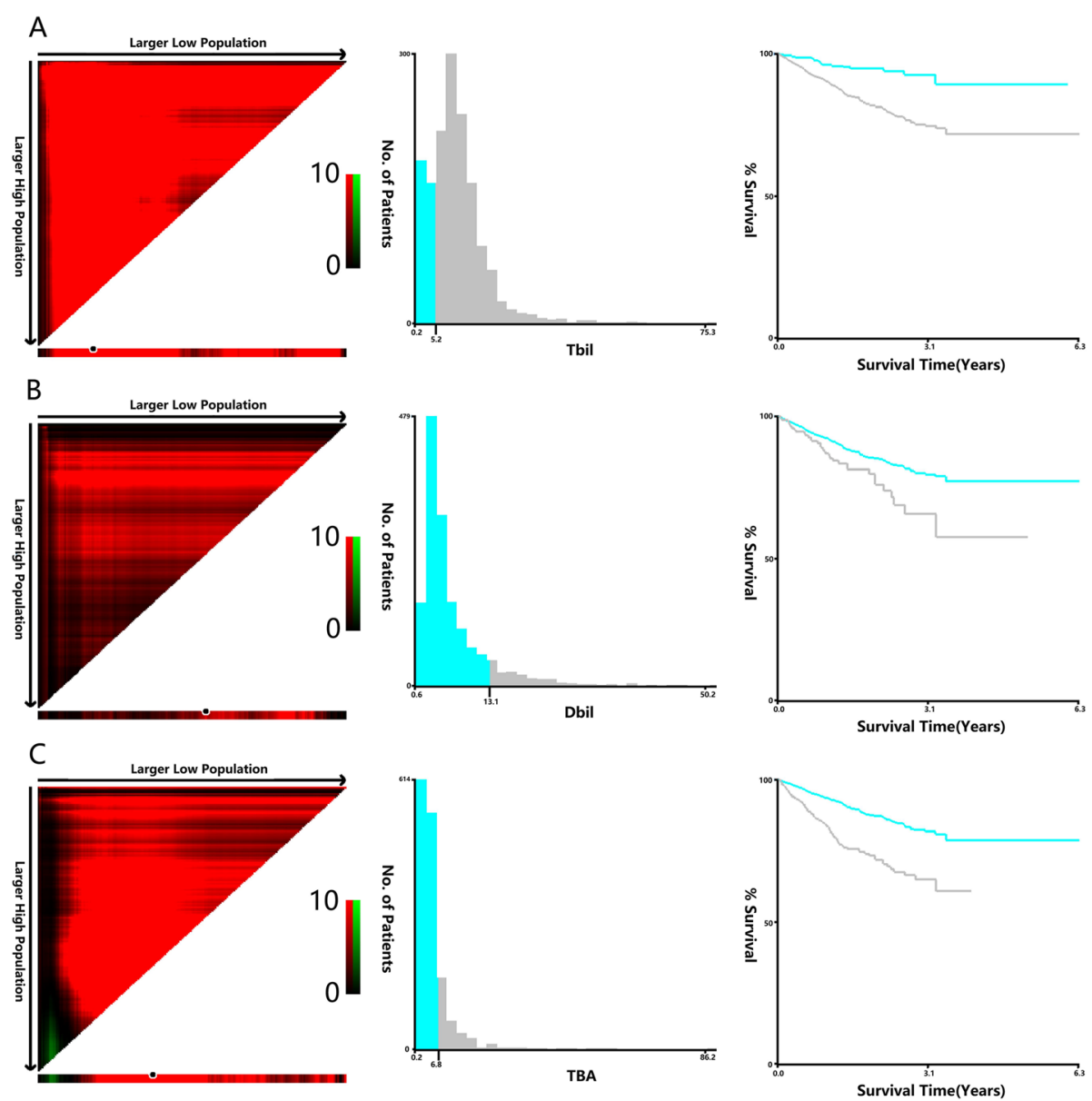

Fig. 1 X-tile analyses of 3-year OS was performed using patients' data to determine the optimal cut-off value for TBIL, DBIL, and TBA. X-tile analyses of TBIL (a), DBIL (b), and TBA (c) levels in CRC patients. X-tile plots for patients are shown in the left panels; black circles highlight the optimal cutoff values, which are also shown in histograms (middle panels). Kaplan-Meier plots are presented in right panels, and in terms of OS, the best cut-off values of TBIL, DBIL and TBA are $6.4 \mu \mathrm{mol} / \mathrm{l}, 12.8 \mathrm{\mu mol} / \mathrm{l}$ and $7.1 \mu \mathrm{mo} / \mathrm{l}$, respectively 

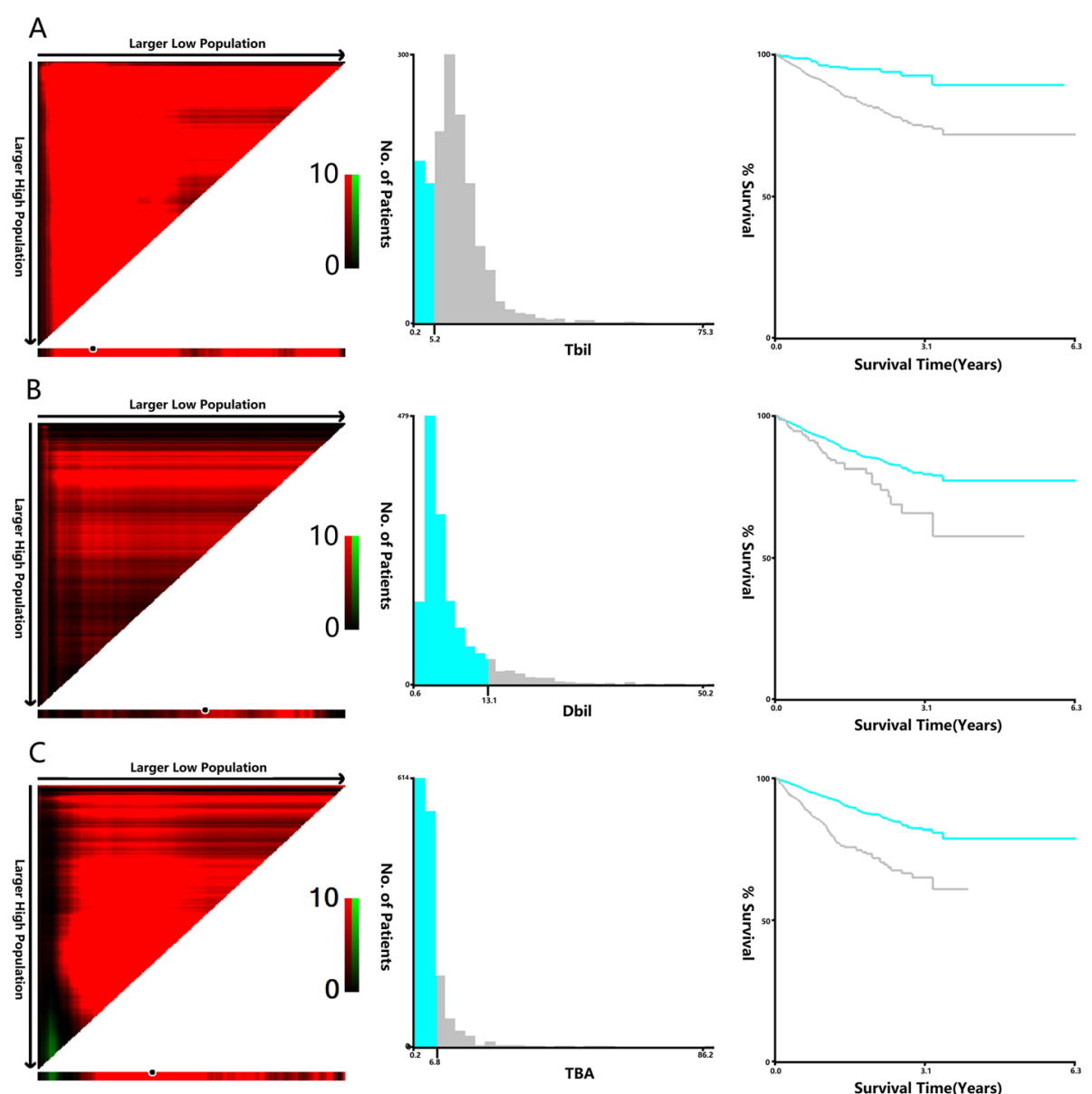

Fig. 2 X-tile analyses of 3-year DFS was performed using patients' data to determine the optimal cut-off value for TBIL, DBIL, and TBA. X-tile analyses of TBIL (a), DBIL (b), and TBA (c) levels in CRC patients. X-tile plots for patients are shown in the left panels; black circles highlight the optimal cutoff values, which are also shown in histograms (middle panels). Kaplan-Meier plots are presented in right panels, and in terms of DFS, the best cut-off values of TBIL, DBIL and TBA are $5.2 \mu \mathrm{mol} / \mathrm{l}, 13.1 \mu \mathrm{mol} / \mathrm{I}$ and $6.8 \mu \mathrm{mol} / \mathrm{l}$, respectively

common characteristics in patients with high DBIL (Table 2). Patients with stage III and stage IV disease were more commonly in the high TBA group than in the low TBA group $(57.4 \%$ vs $51.5 \%, P=0.007)$, and compared to the low TBA group, the high TBA group had significantly more male patients $(64.4 \%$ vs $57.6 \%$, $P=0.045)$ (Table 3).

\section{Prognostic value of TBIL, DBIL and TBA levels}

Patients in the smoking group $(P=0.002)$, with obstruction $(P<0.001)$, colon cancer $(P=0.002)$, advanced $\mathrm{T}$ stage $(P<0.001)$ and $\mathrm{N}$ stage $(P<0.001)$, without chemotherapy $(P<0.001)$, and with high TBIL $(P<0.001)$, DBIL $(P<0.001)$, and TBA $(P<0.001)$ exhibited a worse 3 -year OS (Table 4). In addition, patients in the smoking group $(P<0.001)$, with obstruction $(P<0.001)$, left colon cancer $(P=0.009)$, advanced $\mathrm{T}$ stage $(P<0.001)$ and $\mathrm{N}$ stage $(P<0.001)$, without chemotherapy $(P<0.001)$, with high TBIL $(P<0.001)$, DBIL $(P=0.001)$, and TBA $(P<$ 0.001 ) experienced a shorter 3-year DFS (Table 5).

Next, multivariate Cox regression analysis was performed on the clinical features that were significant in the univariate log-rank test. Results confirmed that TBIL (hazard ratio (HR): 0.436 , confidence interval (CI): 0.329-0.578, $P<0.001$; HR: 0.437, CI: 0.292-0.655, $P<$ 0.001), DBIL (HR: 0.4335, CI: 0.299-0.637, $P<0.001$; HR: 0.583, CI: $0.391-0.871, P=0.008$ ), and TBA (HR: 0.206, CI: 0.124-0.341, $P<0.001$; HR: 0.634, CI: $0.465-$ $0.865, P=0.004$ ) levels were independent prognostic factors for both OS and DFS in patients with CRC after surgical resection (Tables 4 and 5).

\section{Nomogram for predicting CRC outcomes}

To further evaluate the predictive ability of pretreatment levels of serum bilirubin and TBA in CRC, two nomograms were established by a multivariate Cox regression model based on all the significant independent factors 
Table 1 Associations between TBIL level and clinical characteristics in CRC patients

\begin{tabular}{|c|c|c|c|c|c|}
\hline \multirow[t]{2}{*}{ Variable } & & \multicolumn{2}{|l|}{ TBIL group } & \multirow[t]{2}{*}{ Statistics } & \multirow[t]{2}{*}{$P$} \\
\hline & & Low DBIL (n), \% & High DBIL (n), \% & & \\
\hline \multirow[t]{2}{*}{ Sex } & Male & $239(56.5)$ & $628(59.8)$ & 1.316 & 0.251 \\
\hline & Femal & $184(43.5)$ & $423(40.2)$ & & \\
\hline Age & & $57.68 \pm 12.51$ & $58.23 \pm 12.05$ & -0.776 & 0.438 \\
\hline \multirow[t]{2}{*}{ Somking } & Yes & $91(21.5)$ & $212(20.2)$ & 0.332 & 0.564 \\
\hline & No & 332 (778.5) & 839 (79.8) & & \\
\hline \multirow[t]{2}{*}{ Tumor history } & Yes & $43(10.2)$ & $113(10.8)$ & 0.11 & 0.741 \\
\hline & No & $380(79.8)$ & 938 (89.2) & & \\
\hline \multirow[t]{2}{*}{ Obstruction } & Yes & $68(16.1)$ & $170(16.2)$ & 0.002 & 0.963 \\
\hline & No & 355 (83.9) & 881 (83.8) & & \\
\hline Tumor size (cm) & & $4.42 \pm 2.02$ & $4.09 \pm 2.11$ & 2.641 & 0.008 \\
\hline \multirow[t]{3}{*}{ Tumor location } & Right colon & $138(32.6)$ & $313(29.8)$ & 2.791 & 0.248 \\
\hline & Left colon & $88(20.8)$ & $198(18.8)$ & & \\
\hline & Rectum & $197(46.6)$ & $540(51.4)$ & & \\
\hline \multirow[t]{3}{*}{ Differentiation (\%) } & Well & $56(13.2)$ & 165 (15.7) & 1.445 & 0.486 \\
\hline & Moderate & $339(80.1)$ & $820(78.0)$ & & \\
\hline & Poor & $28(6.6)$ & $66(6.3)$ & & \\
\hline \multirow[t]{4}{*}{ T stage (\%) } & $\mathrm{T} 1$ & $36(13.3)$ & $72(6.9)$ & 3.926 & 0.27 \\
\hline & $\mathrm{T} 2$ & $70(16.5)$ & $169(16.1)$ & & \\
\hline & $\mathrm{T} 3$ & $232(54.8)$ & $553(52.6)$ & & \\
\hline & T4 & $85(20.1)$ & $257(24.5)$ & & \\
\hline \multirow[t]{3}{*}{ N stage (\%) } & No & 265 (62.6) & $579(55.1)$ & 7.338 & 0.026 \\
\hline & $\mathrm{N} 1$ & $91(21.5)$ & $284(27.0)$ & & \\
\hline & N2 & 67 (15.8) & $188(17.9)$ & & \\
\hline \multirow[t]{4}{*}{ TNM stage (\%) } & । & $58(13.7)$ & $139(13.2)$ & 2.973 & 0.096 \\
\hline & $\|$ & 155 (36.6) & 347 (33.0) & & \\
\hline & III & $162(38.3)$ & 418 (39.8) & & \\
\hline & IV & $48(11.3)$ & $147(14.0)$ & & \\
\hline \multirow[t]{2}{*}{ Perineural invasion(\%) } & Yes & $94(22.2)$ & $225(21.4)$ & 0.118 & 0.731 \\
\hline & No & $329(77.8)$ & $826(78.6)$ & & \\
\hline \multirow[t]{2}{*}{ Vascular invasion (\%) } & Yes & 77 (18.2) & $188(17.9)$ & 0.02 & 0.887 \\
\hline & No & $346(81.8)$ & $863(82.1)$ & & \\
\hline \multirow[t]{2}{*}{ Chemotherapy (\%) } & Yes & $226(18.2)$ & $556(52.9)$ & 0.033 & 0.855 \\
\hline & No & 197 (46.6) & $495(47.1)$ & & \\
\hline
\end{tabular}

TBIL total bilirubin, CRC Colorectal Cancer

for OS and DFS (Fig. 3a, b). Somke, obstruction, T, N, and $M$ stage, chemotherapy, TBIL, DBIL, and TBA level were included in both prediction models. Nomograms can be created by adding up the scores assigned to each variable, which is indicated at the top of the scale, and the total points can be converted to the predicted 5-year probability of death and recurrence or metastasis for a patient on the lowest scale [11]. Harrell's C-indexes for OS and DFS prediction were 0.819 (95\% CI: $0.806-$ 0.832 ) and 0.835 (95\% CI: 0.822-0.849), respectively, and the calibration curves of the two nomograms showed no deviation from the baseline and no need for recalibration (Fig. 4a, b).

\section{Discussion}

The prognosis of colorectal cancer patients is currently focused on postoperative metastasis and local recurrence of colorectal cancer. At present, there are many studies on prognostic factors in colorectal cancer patients. The primary factors affecting colorectal cancer prognosis 
Table 2 Associations between DBIL level and clinical characteristics in CRC patients

\begin{tabular}{|c|c|c|c|c|c|}
\hline \multirow[t]{2}{*}{ Variable } & & \multicolumn{2}{|l|}{ DBIL group } & \multirow[t]{2}{*}{ Statistics } & \multirow[t]{2}{*}{$P$} \\
\hline & & Low DBIL (n), \% & High DBIL (n), \% & & \\
\hline \multirow[t]{2}{*}{ Sex } & Male & $757(57.7)$ & $110(67.5)$ & 5.681 & 0.017 \\
\hline & Femal & $554(42.3)$ & $53(32.5)$ & & \\
\hline Age & & $58.11 \pm 12.16$ & $54.74 \pm 12.38$ & 0.37 & 0.711 \\
\hline \multirow[t]{2}{*}{ Somking } & Yes & $269(28.1)$ & $34(20.8)$ & 0.01 & 0.919 \\
\hline & No & $1042(71.9)$ & 129 (79.2) & & \\
\hline \multirow[t]{2}{*}{ Tumor history } & Yes & $139(10.6)$ & $17(10.4)$ & 0.005 & 0.946 \\
\hline & No & $1172(89.4)$ & $146(79.6)$ & & \\
\hline \multirow[t]{2}{*}{ Obstruction } & Yes & $202(15.4)$ & $36(22.1)$ & 4.775 & 0.029 \\
\hline & No & 1109 (84.6) & $127(77.9)$ & & \\
\hline Tumor size (cm) & & $4.25 \pm 2.12$ & $3.73 \pm 1.76$ & 2.98 & 0.003 \\
\hline \multirow[t]{3}{*}{ Tumor location } & Right colon & 398 (30.3) & $53(32.5)$ & 0.321 & 0.852 \\
\hline & Left colon & 255 (19.5) & 31 (19.0) & & \\
\hline & Rectum & $658(50.2)$ & 79 (48.5) & & \\
\hline \multirow[t]{3}{*}{ Differentiation (\%) } & Well & $197(15.0)$ & $24(14.7)$ & 3.455 & 0.178 \\
\hline & Moderate & 1025 (78.2) & $134(82.2)$ & & \\
\hline & Poor & $89(6.8)$ & $5(3.1)$ & & \\
\hline \multirow[t]{4}{*}{ T stage (\%) } & $\mathrm{T} 1$ & $93(7.1)$ & $15(9.2)$ & 5.538 & 0.136 \\
\hline & $\mathrm{T} 2$ & $204(15.5)$ & 35 (21.5) & & \\
\hline & T3 & 709 (54.1) & 76 (46.6) & & \\
\hline & T4 & $305(23.3)$ & $37(22.7)$ & & \\
\hline \multirow[t]{3}{*}{ N stage (\%) } & NO & 755 (57.6) & 89 (54.6) & 0.81 & 0.667 \\
\hline & N1 & $333(25.4)$ & $42(25.7)$ & & \\
\hline & N2 & $223(17.0)$ & 32 (19.7) & & \\
\hline \multirow[t]{4}{*}{ TNM stage (\%) } & I & $168(12.9)$ & $29(17.8)$ & 9.65 & 0.022 \\
\hline & ॥ & $463(35.3)$ & 39 (23.9) & & \\
\hline & III & $511(38.9)$ & $69(42.3)$ & & \\
\hline & IV & $169(12.9)$ & $26(16.0)$ & & \\
\hline \multirow[t]{2}{*}{ Perineural invasion (\%) } & Yes & $280(21.4)$ & 39 (23.9) & 0.564 & 0.453 \\
\hline & No & 1031 (78.6) & $124(76.1)$ & & \\
\hline \multirow[t]{2}{*}{ Vascular invasion (\%) } & Yes & $233(17.7)$ & 32 (19.6) & 0.34 & 0.56 \\
\hline & No & $1078(82.3)$ & $131(80.4)$ & & \\
\hline \multirow[t]{2}{*}{ Chemotherapy (\%) } & Yes & 703 (53.6) & $79(48.5)$ & 1.548 & 0.213 \\
\hline & No & $608(46.4)$ & $84(51.5)$ & & \\
\hline
\end{tabular}

DBIL Direct Bilirubin, CRC Colorectal Cancer

include clinicopathology and gene and immune factors [12]. TNM stage, perineural invasion, vascular invasion, chemotherapy, histologic grade, number of resected lymph nodes, lymphatic status, neutrophil-tolymphocyte ratio and platelet-to-lymphocyte ratio have been confirmed as prognostic factors for colorectal cancer $[13,14]$. Other studies have confirmed that colorectal prognosis is closely related to patients' lifestyle, economic status and educational background, such as low education, inappropriate socioeconomic status, and high tumour grade, smoking, and diabetes mellitus conveyed poor survival of CRC patients [15, 16]. In addition, in this study, we confirmed the prognostic significance of serum TBIL, DBIL and TBA levels in colorectal cancer, revealing that elevated TBIL, DBIL and TBA levels were associated with tumour progression and were independent prognostic factors for colorectal cancer patients. Our nomogram also confirmed the prognostic significance of TBIL, DBIL and TBA in patients with colorectal cancer. 
Table 3 Associations between TBA level and clinical characteristics in CRC patients

\begin{tabular}{|c|c|c|c|c|c|}
\hline \multirow[t]{2}{*}{ Variable } & & \multicolumn{2}{|l|}{ TBA group } & \multirow[t]{2}{*}{ Statistics } & \multirow[t]{2}{*}{$\mathbf{P}$} \\
\hline & & Low TBA (n), \% & High TBA (n), \% & & \\
\hline \multirow[t]{2}{*}{ Sex } & Male & $699(57.6)$ & $168(64.4)$ & 4.031 & 0.045 \\
\hline & Femal & $514(42.4)$ & 93 (35.6) & & \\
\hline Age & & $58.11 \pm 12.14$ & $57.87 \pm 12.41$ & 0.287 & 0.774 \\
\hline \multirow[t]{2}{*}{ Somking } & Yes & $246(20.3)$ & 57 (21.8) & 0.32 & 0.572 \\
\hline & No & $967(79.7)$ & $204(78.2)$ & & \\
\hline \multirow[t]{2}{*}{ Tumor history } & Yes & $134(11.1)$ & $22(8.4)$ & 1.555 & 0.212 \\
\hline & No & 1079 (88.9) & 239 (91.6) & & \\
\hline \multirow[t]{3}{*}{ Obstruction } & Obstruction & & & 1.617 & 0.203 \\
\hline & Yes & $189(15.6)$ & 49 (18.8) & & \\
\hline & No & $1024(84.4)$ & $212(81.2)$ & & \\
\hline Tumor size (cm) & & $4.22 \pm 2.12$ & $4.07 \pm 1.93$ & 1.068 & 0.286 \\
\hline \multirow[t]{3}{*}{ Tumor location } & Right colon & $363(29.9)$ & $88(33.7)$ & 1.893 & 0.388 \\
\hline & Left colon & $234(19.3)$ & 52 (19.9) & & \\
\hline & Rectum & $616(50.8)$ & $121(46.4)$ & & \\
\hline \multirow[t]{3}{*}{ Differentiation (\%) } & Well & 176 (14.5) & 45 (17.2) & 2.653 & 0.265 \\
\hline & Moderate & $955(78.7)$ & $204(78.2)$ & & \\
\hline & Poor & $82(6.8)$ & $12(4.6)$ & & \\
\hline \multirow[t]{4}{*}{ T stage (\%) } & $\mathrm{T} 1$ & $92(7.6)$ & $16(6.1)$ & 6.484 & 0.09 \\
\hline & $\mathrm{T} 2$ & $204(16.8)$ & $35(13.4)$ & & \\
\hline & T3 & 650 (53.6) & $135(51.7)$ & & \\
\hline & T4 & $267(22.0)$ & $75(28.7)$ & & \\
\hline \multirow[t]{3}{*}{ N stage (\%) } & NO & 703 (58.0) & $141(54.0)$ & 1.449 & 0.484 \\
\hline & $\mathrm{N} 1$ & $305(25.1)$ & 70 (26.8) & & \\
\hline & N2 & $205(16.9)$ & $50(19.2)$ & & \\
\hline \multirow[t]{4}{*}{ TNM stage (\%) } & I & $170(14.0)$ & $27(10.3)$ & 12.202 & 0.007 \\
\hline & $\|$ & 418 (34.5) & $84(32.2)$ & & \\
\hline & III & 481 (39.7) & 99 (37.9) & & \\
\hline & IV & $144(11.8)$ & $51(19.5)$ & & \\
\hline \multirow[t]{2}{*}{ Perineural invasion (\%) } & Yes & $264(21.8)$ & $55(21.1)$ & 0.061 & 0.806 \\
\hline & No & $949(78.2)$ & $206(78.9)$ & & \\
\hline \multirow[t]{2}{*}{ Vascular invasion (\%) } & Yes & $213(17.6)$ & $52(19.9)$ & 0.814 & 0.367 \\
\hline & No & $1000(82.4)$ & $209(80.1)$ & & \\
\hline \multirow[t]{2}{*}{ Chemotherapy (\%) } & Yes & $657(54.2)$ & $125(47.9)$ & 3.391 & 0.066 \\
\hline & No & $556(45.8)$ & 136 (52.1) & & \\
\hline
\end{tabular}

TBA Total Bile Acid, CRC Colorectal Cancer

Bile acids are an important component of the gastrointestinal tract. They connect the intestinal microbiota with liver and intestinal metabolism, affecting gastrointestinal motility, intestinal permeability and carcinogenesis. Disruption of bile acid-microbiota crosstalk promotes inflammation and the phenotype of gastrointestinal disease, which may contribute to the development of gastrointestinal cancers, including colorectal cancer and hepatocellular carcinoma [17]. Bile acids are now thought to be involved in the development of cancer, and human epidemiological and animal studies have shown that colon cancer risk is also associated with faecal bile acid concentrations. Abnormally high levels of bile acids trigger excessive harmful effects on the colonic mucosa, such as DNA oxidative damage, inflammation, and proliferation, markedly promoting CRC progression after the initiation phase [18]. Studies have found that bile acid concentration can be used as a prognostic 
Table 4 Univariate and multivariate analyses of OS significance of serum bilirubin and TBA levels

\begin{tabular}{|c|c|c|c|c|c|c|}
\hline \multirow[t]{2}{*}{ Variable } & \multicolumn{3}{|c|}{ Univariate analysis } & \multicolumn{3}{|c|}{ Multivariate analysis } \\
\hline & $\mathrm{HR}$ & $95 \% \mathrm{Cl}$ & $\mathbf{P}$ & $\mathrm{HR}$ & $95 \% \mathrm{Cl}$ & $\mathbf{P}$ \\
\hline Sex & 1.065 & $0.823-0.232$ & 0.630 & & & \\
\hline Age & 0.994 & $0.984-1.004$ & 0.268 & & & \\
\hline Somking & 1.843 & $1.257-2.703$ & 0.002 & 1.651 & $1.117-2.439$ & 0.012 \\
\hline Tumor history & 0.703 & $0.435-1.137$ & 0.703 & & & \\
\hline Obstruction & 2.456 & $1.861-3.241$ & $<0.001$ & 1.843 & $1.374-2.473$ & $<0.001$ \\
\hline Tumor size & 1.01 & $0.951-1.072$ & 0.748 & & & \\
\hline \multicolumn{7}{|l|}{ Tumor location } \\
\hline Rectum & & Reference & & & Reference & \\
\hline Right colon & 1.574 & $1.18-2.101$ & 0.002 & 1.146 & $0.811-1.619$ & 0.440 \\
\hline Left colon & 1.56 & $1.12-2.171$ & 0.008 & 1.400 & $0.603-1.111$ & 0.199 \\
\hline \multicolumn{7}{|l|}{ Differentiation } \\
\hline Well & & Reference & & & & \\
\hline Moderate & 0.99 & $0.699-1.402$ & 0.954 & & & \\
\hline Poor & 0.646 & $0.322-1.296$ & 0.218 & & & \\
\hline \multicolumn{7}{|l|}{ T stage } \\
\hline $\mathrm{T} 1$ & & Reference & & & Reference & \\
\hline $\mathrm{T} 2$ & 0.875 & $0.375-2.045$ & 0.758 & 0.87 & $0.368-2.058$ & 0.752 \\
\hline T3 & 1.803 & $0.877-3.705$ & 0.109 & 1.775 & $0.828-3.805$ & 0.140 \\
\hline $\mathrm{T} 4$ & 5.968 & $2.915-12.219$ & $<0.001$ & 3.486 & $1.622-7.534$ & 0.001 \\
\hline \multicolumn{7}{|l|}{ N stage } \\
\hline No & & Reference & & & Reference & \\
\hline N1 & 2.068 & $1.512-2.829$ & $<0.001$ & 1.311 & $0.861-1.998$ & 0.207 \\
\hline N2 & 4.149 & $3.063-5.620$ & $<0.001$ & 2.309 & $1.539-3.466$ & $<0.001$ \\
\hline \multicolumn{7}{|l|}{ TNM stage } \\
\hline 1 & & Reference & & & Reference & \\
\hline$\|$ & 1.914 & $0.935-3.914$ & 0.076 & 0.751 & $0.328-1.719$ & 0.498 \\
\hline III & 4.312 & $2.184-8.513$ & $<0.001$ & 1.031 & $0.446-2.384$ & 0.943 \\
\hline IV & 10.482 & $5.259-20.893$ & $<0.001$ & 2.588 & $1.135-5.902$ & 0.024 \\
\hline Perineural invasion & 0.869 & $0.632-1.196$ & 0.390 & & & \\
\hline Vascular invasion & 0.952 & $0.675-1.343$ & 0.781 & & & \\
\hline Chemotherapy & 0.626 & $0.483-0.810$ & $<0.001$ & 0.579 & $0.442-0.76$ & $<0.001$ \\
\hline TBIL & 0.188 & $0.116-0.303$ & $<0.001$ & 0.436 & $0.329-0.578$ & $<0.001$ \\
\hline DBIL & 0.528 & $0.376-0.742$ & $<0.001$ & 0.435 & $0.299-0.637$ & $<0.001$ \\
\hline TBA & 0.26 & $0.201-0.337$ & $<0.001$ & 0.206 & $0.124-0.341$ & $<0.001$ \\
\hline
\end{tabular}

OS Overall Survival, HR Hazard Ratio, CI Confidence Interval, TBIL Total Bilirubin, DBIL Direct Bilirubin, TBA Total Bile Acid

factor in some tumours, primarily because it affects changes in the intestinal flora, leading to the occurrence and development of diseases and contributing to patient prognosis [19]. However, to our knowledge, no studies have reported that levels of total bile acids in patients are an independent prognostic factor in colorectal cancer. In our study, X-Tile software was used to determine the optimal cut-off values of $7.1 \mu \mathrm{mol} / \mathrm{L}$ for TBA for 3year OS and $6.8 \mu \mathrm{mol} / \mathrm{L}$ for TBA for 3-year DFS. In the clinicopathological characteristics univariate analysis, the high TBA group had a higher percentage of TNM stage (III and IV) than the low TBA group $(P=0.007)$. Multivariate Cox regression analyses confirmed that a high TBA level was an independent prognostic factor for both OS and DFS $(p<0.05)$.

Bile acids are the final product of cholesterol decomposition. They occur in hepatocytes and peripheral blood in three forms: TBIL, DBIL and IDBIL. In addition to 
Table 5 Univariate and multivariate analyses of DFS significance of serum bilirubin and TBA levels

\begin{tabular}{|c|c|c|c|c|c|c|}
\hline \multirow[t]{2}{*}{ Variable } & \multicolumn{3}{|c|}{ Univariate analysis } & \multicolumn{3}{|c|}{ Multivariate analysis } \\
\hline & HR & $95 \% \mathrm{Cl}$ & $\mathbf{P}$ & $\mathrm{HR}$ & $95 \% \mathrm{Cl}$ & $\mathbf{P}$ \\
\hline Sex & 0.928 & $0.711-1.212$ & 0.583 & & & \\
\hline Age & 0.995 & $0.985-1.006$ & 0.379 & & & \\
\hline Somking & 2.322 & $1.495-3.607$ & $<0.001$ & 1.851 & $1.184-2.894$ & 0.007 \\
\hline Tumor history & 0.949 & $0.605-1.488$ & 0.819 & & & \\
\hline Obstruction & 0.504 & $0.375-0.678$ & $<0.001$ & 0.576 & $0.423-0.786$ & $<0.001$ \\
\hline Tumor size & 0.990 & $0.928-1.055$ & 0.750 & & & \\
\hline \multicolumn{7}{|l|}{ Tumor location } \\
\hline Rectum & & Reference & & & Reference & \\
\hline Right colon & 0.880 & $0.614-1.263$ & 0.489 & 1.191 & $0.871-1.629$ & 0.275 \\
\hline Left colon & 0.672 & $0.499-0.904$ & 0.009 & 1.333 & $0.915-1.942$ & 0.135 \\
\hline \multicolumn{7}{|l|}{ Differentiation } \\
\hline Well & & Reference & & & & \\
\hline Moderate & 1.284 & $0.862-1.913$ & 0.219 & & & \\
\hline Poor & 1.288 & $0.688-2.413$ & 0.428 & & & \\
\hline T stage & & & & & & 0.004 \\
\hline $\mathrm{T} 1$ & & Reference & & & Reference & \\
\hline $\mathrm{T} 2$ & 0.128 & $0.052-0.314$ & $<0.001$ & 1.603 & $0.58-4.429$ & 0.363 \\
\hline T3 & 0.177 & $0.105-0.3$ & $<0.001$ & 2.361 & $0.94-5.932$ & 0.067 \\
\hline $\mathrm{T} 4$ & 0.357 & $0.27-0.471$ & $<0.001$ & 3.431 & $1.353-8.704$ & 0.009 \\
\hline N stage & & & & & & 0.008 \\
\hline No & & Reference & & & Reference & \\
\hline N1 & 1.906 & $1.37-2.651$ & $<0.001$ & 1.120 & $0.75-1.673$ & 0.580 \\
\hline $\mathrm{N} 2$ & 4.020 & $2.941-5.495$ & $<0.001$ & 1.696 & $1.167-2.466$ & 0.006 \\
\hline TNM stage & & & & & & $<0.001$ \\
\hline I & & Reference & & & Reference & \\
\hline$\|$ & 2.242 & $0.87-5.778$ & 0.095 & 1.180 & $0.418-3.33$ & 0.754 \\
\hline III & 4.968 & $2.008-12.295$ & 0.001 & 1.980 & $0.713-5.497$ & 0.190 \\
\hline IV & 28.627 & $11.687-70.12$ & $<0.001$ & 10.452 & $3.842-28.433$ & $<0.001$ \\
\hline Perineural invasion & 1.343 & $0.976-1.846$ & 0.070 & & & \\
\hline Vascular invasion & 1.165 & $0.826-1.643$ & 0.385 & & & \\
\hline Chemotherapy & 0.950 & $0.729-1.237$ & 0.702 & & & \\
\hline TBIL & 0.390 & $0.267-0.57$ & $<0.001$ & 0.437 & $0.292-0.655$ & $<0.001$ \\
\hline DBIL & 0.618 & $0.429-0.891$ & 0.010 & 0.583 & $0.391-0.871$ & 0.008 \\
\hline TBA & 0.408 & $0.308-0.541$ & $<0.001$ & 0.634 & $0.465-0.865$ & 0.004 \\
\hline
\end{tabular}

DFS Disease Free Survival, HR Hazard Ratio, Cl Confidence Interval, TBIL Total Bilirubin, DBIL Direct Bilirubin, TBA Total Bile Acid

total bile acids being considered an independent prognostic factor for colorectal cancer, our results showed that TBIL and DBIL were also independent prognostic factors. The high DBIL group had a higher percentage of obstruction and TNM stage (III and IV) compared to the high DBIL group $(P=0.029, P=0.022)$, and patients in the low DBIL had larger tumour size than those in the high DBIL group $(P=0.003)$. The high TBIL group exhibited a higher percentage of lymph node metastasis
(N1 and N2) compared to the low TBIL group ( $P=$ 0.026), and patients in the low TBIL exhibited larger tumour size than those in the high TBIL group $(P=$ 0.008). Multivariate Cox regression analyses confirmed that high DBIL and TBIL levels were independent prognostic factors for both 3-year OS and DFS. At present, similar results have been obtained regarding the prognostic effect of serum bilirubin levels on malignant tumours. Zhang et al. found that high DBIL level was an 


\section{A}

Points

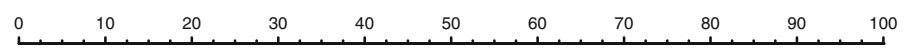

Smoke

T stage

TNM stage

$\mathrm{N}$ stage

Obstruction

TBA

Tbil
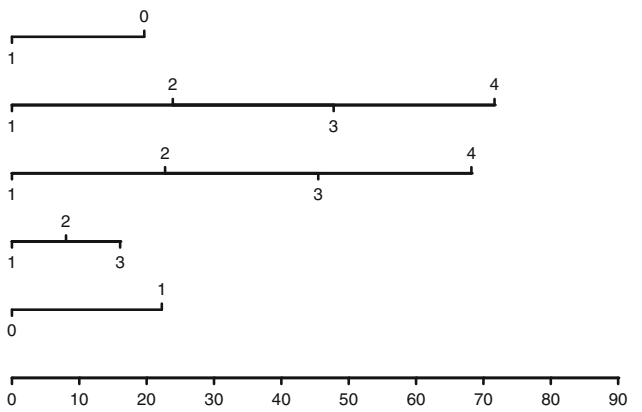

Dbil

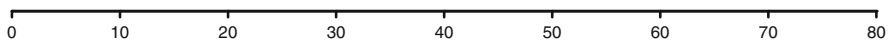

Adjuvant

chemotherapy

Total Points

\begin{tabular}{lllllll}
\hline & 10 & 20 & 30 & 40 & 50
\end{tabular}

3-year overall survival

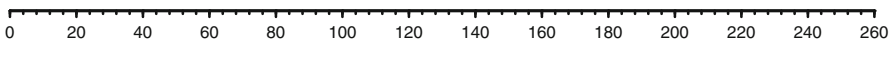

-year overall survival

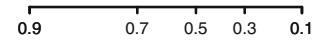

B

Points

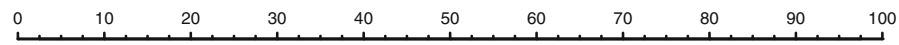

Smoke

T stage

$\mathrm{N}$ stage

Obstruction

TNM stage

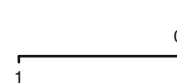

0

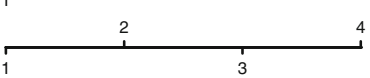

$\stackrel{2}{2}$
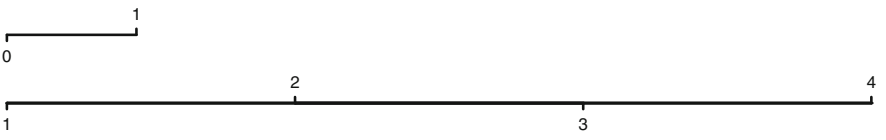

TBA

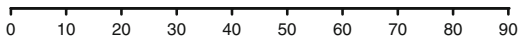

Tbil

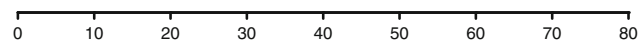

Dbil

Total Points

\begin{tabular}{llllllll}
\hline & 5 & 15 & 25 & 35 & 45 & 55
\end{tabular}

Linear Predictor

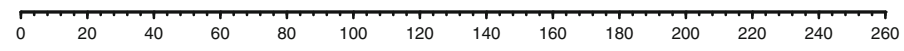

3-year disease-free survival

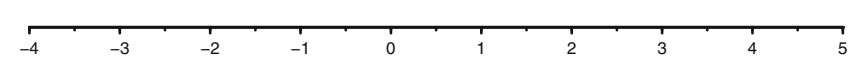

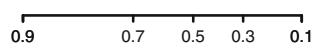

Fig. 3 Nomogram for predicting CRC patient outcomes. Nomograms conveyed the results of prognostic models using clinicopathological characteristics and pretreatment inflammatory biomarkers to predict OS (a) and DFS (b) of patients with CRC. The Harrell's c-indexes for OS and DFS prediction were 0.819 (95\% Cl: 0.806-0.832) and 0.835 (95\% Cl: 0.822-0.849), respectively

independent prognostic factor for OS and DFS, and a higher proportion of lymph node metastasis and lymphovascular invasion was observed at higher DBIL levels than at lower DBIL levels [10]. Yang et al. also found that in stage IV CRC patients, elevated levels of TBIL and DBIL were associated with poor OS [20]. DBIL can be considered an independent prognostic biomarker of OS, and the prognostic effect of DBIL on OS is similar to that of carcinoembryonic antigen (CEA). Bilirubin levels have been associated with the risk of several malignancies. Studies have found that moderately elevated pretreatment bilirubin levels are associated with longer 


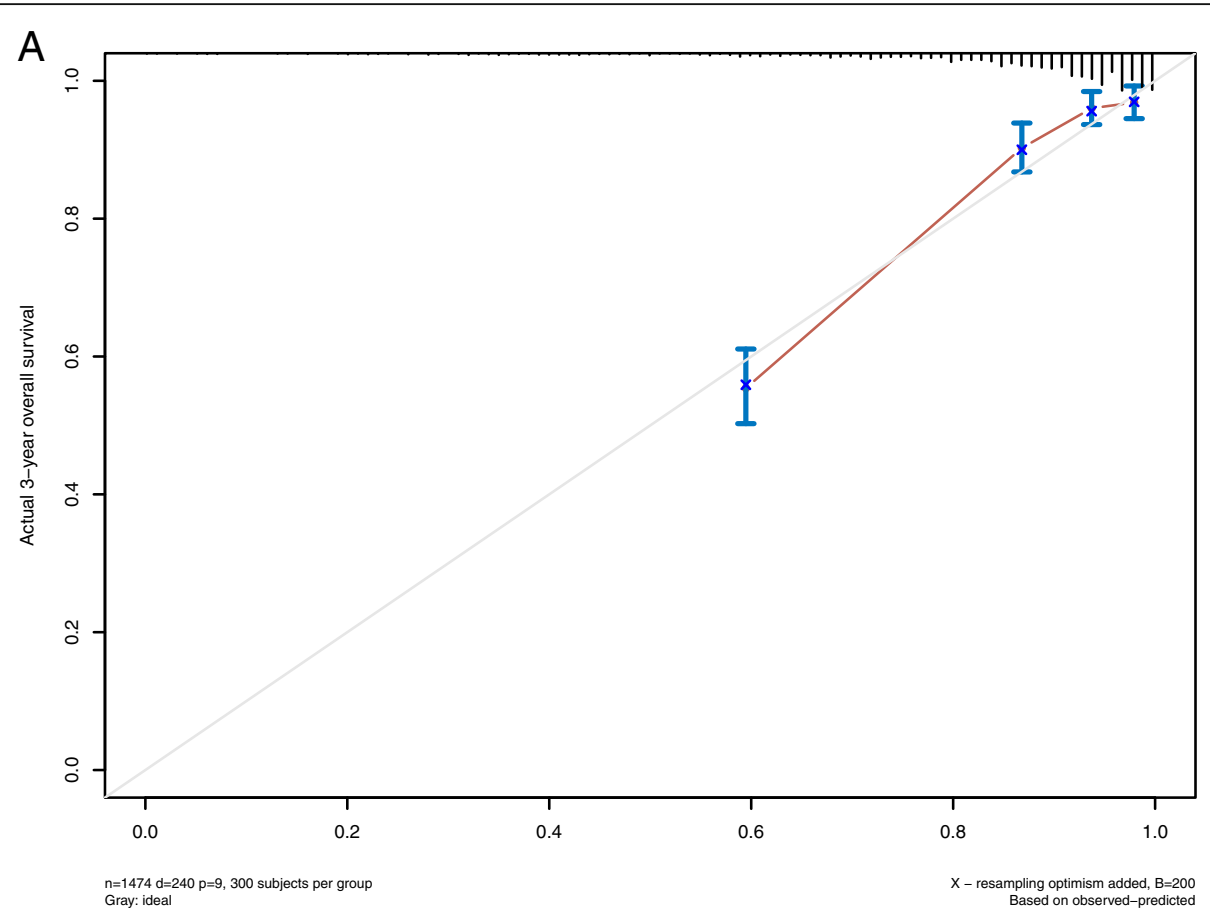

B

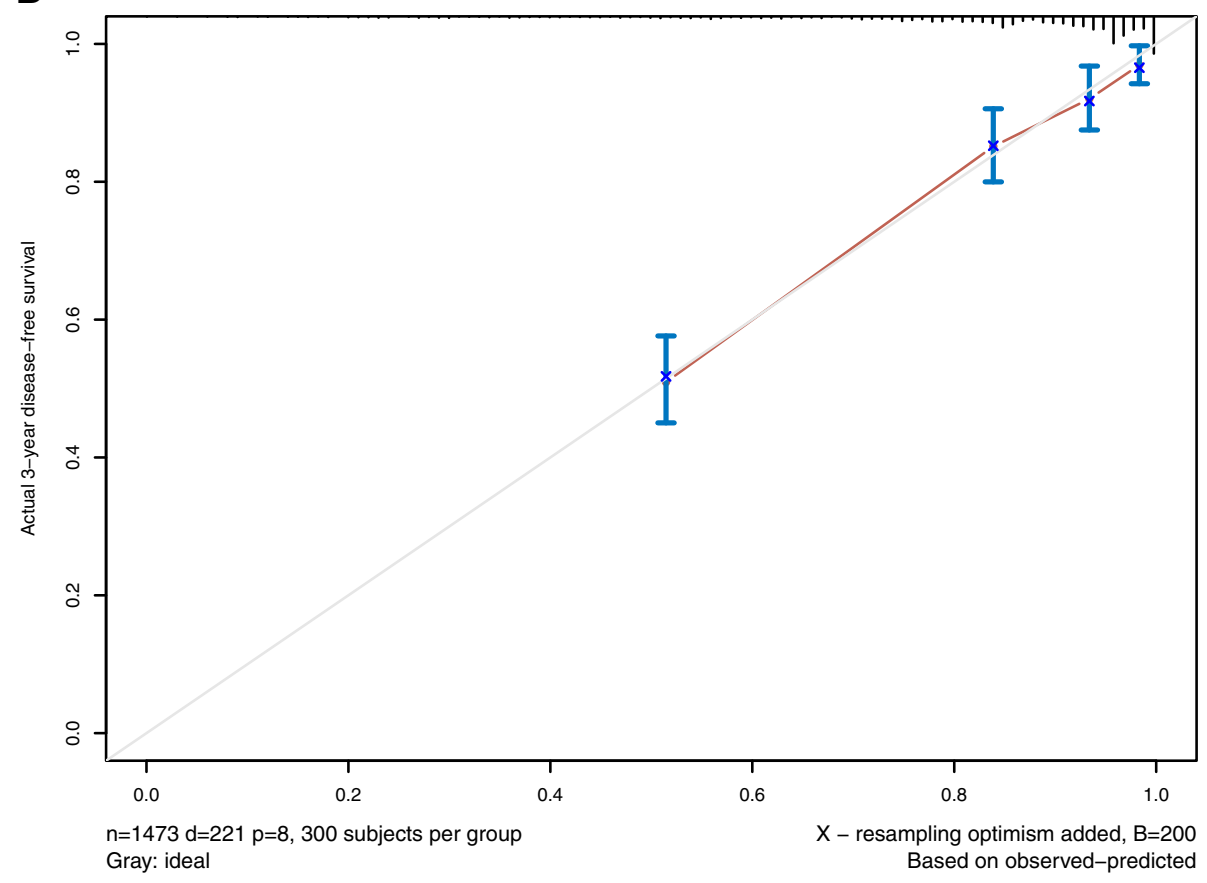

Fig. 4 Calibration curves. Calibration curves for 3-year OS (a) and 3-year DFS (b) using nomograms with clinicopathological characteristics and pretreatment inflammatory biomarkers are shown. The 45-degree reference line represents a perfect match between observed and predicted values

OS and DFS in patients with non-small cell lung cancer [21]. A cross-sectional study found that TBIL and DBIL in patients with gastric cancer were significantly lower compared to healthy controls. The effect of bilirubin level on prognosis was similar to CEA and carbohydrate antigen (CA) 19-9 [22]. Sun et al. found that TBIL and albumin levels are independent predictors of OS in patients with gastric cancer, and the combination of TBIL and albumin levels with TNM staging system indicators is of greater prognostic value [23]. Other studies have found that serum bilirubin in patients with advanced pancreatic cancer, TBIL and DBIL are independent of 
patient OS and are not a prognostic factor [24]. The prognostic significance of serum bilirubin is inconsistent among different types of tumours, and further studies are needed to determine whether pretreatment levels of serum bilirubin is a protective or harmful prognostic factor in CRC.

Although serum markers are an important factor in the prognosis of cancer patients, a single marker may not be sufficient to predict survival in a clinical setting. Combining multiple markers in one index can improve their predictive ability [25-28]. Nomograms combine clinical characteristics to improve the accuracy of survival prediction. In the present study, we constructed a nomogram based on clinicopathological characteristics and pretreatment inflammatory biomarkers (smoking, $\mathrm{T}$ stage, N stage, TNM staging, obstruction, TBIL, DBIL, adjuvant chemotherapy) to predict 3-year OS and DFS in CRC patients. Harrell's C-index confirms the accuracy of these predictions. Harrell's C-indexes for OS and DFS prediction were 0.819 (95\% CI: $0.806-0.832)$ and 0.835 (95\% CI: 0.822-0.849), respectively. These nomograms based on OS and DFS can be used as a practical model for evaluating prognosis in CRC patients.

Our research has some limitations. First, studies have found that the ratio of serum bilirubin to albumin is an important prognostic factor for patients with colorectal cancer with liver metastasis [29]. However, in our study, due to the lack of data from some patients with colorectal cancer with liver metastasis, we did not conduct a separate analysis of these patients, which may lead to errors in the results, and further research will clarify this issue. Second, since a retrospective design was used in this study, there may be confounders affecting the results. Next, this study only analysed the serum levels of bilirubin and bile acid in patients before treatment and was unable to investigate changes in these indicators during treatment or whether these changes affected survival outcomes in CRC patients. Finally, our study was a single-centre retrospective study, and a multicentre study with longer follow-up is needed to verify whether our findings are universally applicable. Nevertheless, several significant advantages of this study include a relatively large retrospective cohort analysis (large sample size) and a broad meta-analysis of various factors that may be associated with colorectal cancer OS and DFS, which should provide an important reference point for clinicians.

\section{Conclusions}

This is the first report to demonstrate the combination of pretreatment levels of serum bilirubin and TBA levels to predict survival outcomes in CRC patients after radical resection in China. Our retrospective study revealed that serum TBIL, DBIL and TBA before treatment were significantly correlated with CRC patient prognosis and were independent prognostic factors. The nomograms based on OS and DFS can be used as a practical model for evaluating the prognosis of CRC patients. This biomarker is directly derived from routine laboratory tests of liver function and can be easily applied in clinical practice. Further study and analysis are needed to investigate its prognostic role in malignant tumours of different organs.

\begin{abstract}
Abbreviations
CRC: Colorectal cancer; TBA: Total bile acid; TBIL: Total bilirubin; DBIL: Direct bilirubin; OS: Overall survival; DFS: Disease free survival; HR: Hazard ratio; Cl: Confidence interval; TNM: Tumor-node-metastasis; AJCC: American Joint Committee on Cancer; CA: Carbohydrate antigen; CEA: Carcinoembryonic antigen
\end{abstract}

\section{Acknowledgements}

Not applicable.

\section{Authors' contributions}

All authors have read and approved the manuscript. Y. C, S. D, and L.Y. lead the study. Y.C. performed the data analysis, implemented the methodology; J.G., J.Y., M.Y. collected the data; Y.C. prepared the original draft; Y.C. and L.L. helped to perfect the figures K.C. reviewed and edited the final manuscript.

\section{Funding}

This study was supported by the Graduates' Innovation Fund, Huazhong University of Science and Technology (No. 2020yjsCXCY055); Free innovation pre-research fund and platform scientific research fund in 2019 (02.03.2019111). The funding body had no role in the design of the study and collection, analysis, and interpretation of data and in writing this manuscript.

\section{Availability of data and materials}

The datasets used and/or analysed during the current study are available from the corresponding author on reasonable request.

\section{Ethics approval and consent to participate}

The study ethics approval was granted from the local ethical committee of the Wuhan Union Hospital (No.2018-S377), and the study was performed in accordance with the principles of the Declaration of Helsinki. The recruited volunteers were requested to sign an informed consent form.

\section{Consent for publication}

Not applicable.

\section{Competing interests}

The authors declare that they have no competing interests.

\section{Author details}

${ }^{1}$ Department of Gastrointestinal Surgery, Union Hospital, Tongji Medical College, Huazhong University of Science and Technology, 1277 JieFang Avenue, Wuhan 430022, Hubei, China. ${ }^{2}$ Department of Gastrointestinal Surgery, The Central Hospital of Wuhan, Tongji Medical College, Huazhong University of Science and Technology, Wuhan, China. ${ }^{3}$ Department of Pathology, Union Hospital, Tongji Medical, Huazhong University of Science and Technology, Wuhan 430022, Hubei, China. ${ }^{4}$ Department of Epidemiology and Biostatistics, the Ministry of Education Key Lab of Environment and Health, School of Public Health, Tongji Medical College, Huazhong University of Science and Technology, Wuhan, Hubei 430022, China.

Received: 21 July 2020 Accepted: 11 January 2021

Published online: 21 January 2021

\section{References}

1. Miller KD, Nogueira L, Mariotto AB, Rowland JH, Yabroff KR, Alfano CM, Jemal A, Kramer JL, Siegel RL. Cancer treatment and survivorship statistics, 2019. CA Cancer J Clin. 2019;69(5):363-85. 
2. Da RP. Quality focus: trends in incidence and mortality of early age onset colorectal Cancer in South Dakota. S D Med. 2020;73(3):139.

3. Dikken JL, van de Velde CJ, Gonen M, Verheij M, Brennan MF, Coit DG. The new American joint committee on Cancer/International Union against Cancer staging system for adenocarcinoma of the stomach: increased complexity without clear improvement in predictive accuracy. Ann Surg Oncol. 2012;19(8):2443-51.

4. Shin DW, Kim J. The American joint committee on Cancer 8th edition staging system for the pancreatic ductal adenocarcinoma: is it better than the 7th edition? Hepatobiliary Surg Nutr. 2020;9(1):98-100.

5. Gao C, Fang L, Li JT, Zhao HC. Significance and prognostic value of increased serum direct bilirubin level for lymph node metastasis in Chinese rectal cancer patients. World J Gastroenterol. 2016;22(8):2576-84.

6. Ioannou GN, Liou IW, Weiss NS. Serum bilirubin and colorectal cancer risk: a population-based cohort study. Aliment Pharmacol Ther. 2006;23(11):1637-42.

7. Herbosa CM, Hodges W, Mann C, Demehri S, Cornelius LA, Semenov YR. Risk of cancer in psoriasis: study of a nationally representative sample of the US population with comparison to a single-institution cohort. J Eur Acad Dermatol Venereol. 2020;34(9):e529-e531.

8. Horsfall $\amalg$, Rait G, Walters K, Swallow DM, Pereira SP, Nazareth I, Petersen I. Serum bilirubin and risk of respiratory disease and death. JAMA. 2011;305(7): $691-7$.

9. Luceri C, Femia AP, D'Ambrosio M, Caderni G. High sensitivity to Cholic acid-induced colonic tumorigenesis makes female PIRC rats (F344/NTac-Apc (am1137)) a suitable model for studying CRC-promoting agents. Anticancer Res. 2019;39(9):4673-9.

10. Zhang Q, Ma X, Xu Q, Qin J, Wang Y, Liu Q, Wang H, Li M. Nomograms incorporated serum direct bilirubin level for predicting prognosis in stages II and III colorectal cancer after radical resection. Oncotarget. 2017;8(41): 71138-46.

11. Li Y, Jia H, Yu W, Xu Y, Li X, Li Q, Cai S. Nomograms for predicting prognostic value of inflammatory biomarkers in colorectal cancer patients after radical resection. Int J Cancer. 2016;139(1):220-31.

12. Marks KM, West NP, Morris E, Quirke P. Clinicopathological, genomic and immunological factors in colorectal cancer prognosis. Br J Surg. 2018;105(2): e99-e109.

13. Kim JH, Lee JY, Kim HK, Lee JW, Jung SG, Jung K, Kim SE, Moon W, Park MI, Park SJ. Prognostic significance of the neutrophil-to-lymphocyte ratio and platelet-to-lymphocyte ratio in patients with stage III and IV colorectal cancer. World J Gastroenterol. 2017;23(3):505-15.

14. Yamaguchi K, Ogata Y, Akagi Y, Shirouzu K. Identification of high-risk factors as indicators for adjuvant therapy in stage II colon cancer patients treated at a single institution. Oncol Lett. 2013;6(3):659-66.

15. Rasouli MA, Moradi G, Roshani D, Nikkhoo B, Ghaderi E, Ghaytasi B. Prognostic factors and survival of colorectal cancer in Kurdistan province, Iran: a population-based study (2009-2014). Medicine (Baltimore). 2017;96(6): e5941.

16. Croft B, Reed M, Patrick C, Kovacevich N, Voutsadakis IA. Diabetes, obesity, and the metabolic syndrome as prognostic factors in stages I to III colorectal Cancer patients. J Gastrointest Cancer. 2019;50(2):221-9.

17. Jia W, Xie G, Jia W. Bile acid-microbiota crosstalk in gastrointestinal inflammation and carcinogenesis. Nat Rev Gastroenterol Hepatol. 2018;15(2): $111-28$.

18. Gadaleta RM, Garcia-Irigoyen O, Moschetta A. Bile acids and colon cancer: is FXR the solution of the conundrum? Mol Asp Med. 2017;56:66-74.

19. Alexander J. PASA: OC-061 a prospective multi-national study of the colorectal cancer mucosal microbiome reveals specific taxonomic changes indicative of disease stage and prognosis. Gut. 2017;66:A32-3.

20. Yang $L$, Ge $L Y$, Yu T, Liang $Y$, Yin $Y$, Chen $H$. The prognostic impact of serum bilirubin in stage IV colorectal cancer patients. J Clin Lab Anal. 2018;32(2): e22272.

21. Li N, Xu M, Cai MY, Zhou F, Li CF, Wang BX, Ou W, Wang SY. Elevated serum bilirubin levels are associated with improved survival in patients with curatively resected non-small-cell lung cancer. Cancer Epidemiol. 2015;39(5): 763-8.

22. Wei TT, Wang LL, Yin JR, Liu YT, Qin BD, Li JY, Yin X, Zhou L, Zhong RQ. Relationship between red blood cell distribution width, bilirubin, and clinical characteristics of patients with gastric cancer. Int J Lab Hematol. 2017;39(5):497-501.
23. Sun $H$, He B, Nie Z, Pan Y, Lin $K$, Peng H, Xu T, Chen X, Hu X, Wu Z, et al. A nomogram based on serum bilirubin and albumin levels predicts survival in gastric cancer patients. Oncotarget. 2017:8(25):41305-18.

24. Xu YX, Wang YB, Tan YL, Xi C, Xu XZ. Prognostic value of pretreatment albumin to bilirubin ratio in patients with hepatocellular cancer: a metaanalysis. Medicine (Baltimore). 2019;98(2):e14027.

25. Deng Q, He B, Liu X, Yue J, Ying H, Pan Y, Sun H, Chen J, Wang F, Gao T, et al. Prognostic value of pre-operative inflammatory response biomarkers in gastric cancer patients and the construction of a predictive model. J Transl Med. 2015;13:66.

26. Beom SH, Shin SJ, Kim CG, Kim JH, Hur H, Min BS, Lee KY, Kim NK, Ahn JB. Clinical Significance of Preoperative Serum Carcinoembryonic Antigen Within the Normal Range in Colorectal Cancer Patients Undergoing Curative Resection. Ann Surg Oncol. 2020;27(8):2774-83.

27. Camp RL, Dolled-Filhart M, Rimm DL. X-tile: a new bio-informatics tool for biomarker assessment and outcome-based cut-point optimization. Clin Cancer Res. 2004;10(21):7252-9.

28. Ogluszka M, Orzechowska M, Jedroszka D, Witas P, Bednarek AK. Evaluate Cutpoints: adaptable continuous data distribution system for determining survival in Kaplan-Meier estimator. Comput Methods Prog Biomed. 2019; 177:133-9.

29. Abdel-Rahman O. Prognostic value of baseline ALBI score among patients with colorectal liver metastases: a pooled analysis of two randomized trials. Clin Colorectal Cancer. 2019;18(1):e61-8.

\section{Publisher's Note}

Springer Nature remains neutral with regard to jurisdictional claims in published maps and institutional affiliations.
Ready to submit your research? Choose BMC and benefit from:

- fast, convenient online submission

- thorough peer review by experienced researchers in your field

- rapid publication on acceptance

- support for research data, including large and complex data types

- gold Open Access which fosters wider collaboration and increased citations

- maximum visibility for your research: over $100 \mathrm{M}$ website views per year

At BMC, research is always in progress.

Learn more biomedcentral.com/submissions 